\title{
Review
}

\section{Artificial intelligence in medicine}

\author{
AN Ramesh ${ }^{1}$, C Kambhampati², JRT Monson ${ }^{1}$, PJ Drew ${ }^{1}$ \\ ${ }^{1}$ The University of Hull Academic Surgical Unit, Castle Hill Hospital, Cottingham, UK \\ ${ }^{2}$ Department of Computer Science, University of Hull, UK
}

\begin{abstract}
Introduction: Artificial intelligence is a branch of computer science capable of analysing complex medical data. Their potential to exploit meaningful relationship with in a data set can be used in the diagnosis, treatment and predicting outcome in many clinical scenarios.

Methods: Medline and internet searches were carried out using the keywords artificial intelligence' and 'neural networks (computer)'. Further references were obtained by crossreferencing from key articles. An overview of different artificial intelligent techniques is presented in this paper along with the review of important clinical applications.

Results: The proficiency of artificial intelligent techniques has been explored in almost every field of medicine. Artificial neural network was the most commonly used analytical tool whilst other artificial intelligent techniques such as fuzzy expert systems, evolutionary computation and hybrid intelligent systems have all been used in different clinical settings.

Discussion: Artificial intelligence techniques have the potential to be applied in almost every field of medicine. There is need for further clinical trials which are appropriately designed before these emergent techniques find application in the real clinical setting.
\end{abstract}

Key words: Artificial intelligence - Neural networks (computer)

A rtificial intelligence (AI) is defined as 'a field of science and engineering concerned with the computational understanding of what is commonly called intelligent behaviour, and with the creation of artefacts that exhibit such behaviour'. ${ }^{1}$ Aristotle attempted to formalise 'right thinking' (logic) through his syllogisms (a three part deductive reasoning). Much of the work in the modern era was inspired by this and the early studies on the operation of mind helped to establish contemporary logical thinking. Programs which enable computers to function in the ways, that make people seem intelligent are called artificial intelligent systems. The British mathematician Alan Turing (1950) was one of the founders of modern computer science and AI. He defined intelligent behaviour in a computer as the ability to achieve human-level performance in cognitive tasks, this later became popular as the 'Turing test'. ${ }^{2}$ Since the middle of the last century, researchers have explored the potential applications of intelligent techniques in every field of medicine. ${ }^{3,4}$ The application of AI technology in the field of surgery was first successively investigated by Gunn in 1976, when he explored the possibility of diagnosing acute abdominal pain with computer analysis. ${ }^{5}$ The last two decades have seen a surge in the interest in medical AI.

Modern medicine is faced with the challenge of acquiring, analysing and applying the large amount of knowledge necessary to solve complex clinical problems. The development of medical artificial intelligence has been related to the development of AI programs intended

Correspondence to: Mr PJ Drew, Academic Surgical Unit, Castle Hill Hospital, Cottingham HU16 5JQ, UK

Tel: +44 (0) 1482 623077; Fax: +44 (0) 1482 623274; E-mail: P.J.Drew@hull.ac.uk 
to help the clinician in the formulation of a diagnosis, the making of therapeutic decisions and the prediction of outcome. They are designed to support healthcare workers in their every day duties, assisting with tasks that rely on the manipulation of data and knowledge. Such systems include Artificial neural networks (ANNs), fuzzy expert systems, evolutionary computation and hybrid intelligent systems.

\section{Artificial neural networks}

Judging by the volume of publication in the last two decades, ANN is the most popular AI technique in medicine. ${ }^{6}$ ANNs are computational analytical tools which are inspired by the biological nervous system. They consist of networks of highly interconnected computer processors called 'neurons' that are capable of performing parallel computations for data processing and knowledge representation. Their ability to learn from historical examples, analyse non-linear data, handle imprecise information and generalise enabling application of the model to independent data has made them a very attractive analytical tool in the field of medicine.

McCulloch and Pitts (1943) invented the first artificial neurone using simple binary threshold functions. ${ }^{7}$ The next important milestone came when Frank Rosenblatt, a psychologist, developed the Perceptron in $1958^{8}$ as a practical model. Many variations of the basic Perceptron network have been proposed but the most popular model has been multilayer feedforward Perceptron (Fig. 1). These networks are made up of layers of neurons, typically an input layer, one or more middle or hidden layers and an output layer, each of which are fully connected to other layer. The neurons are connected by links, and each link has a numerical weight associated with it. A neural network 'learns' through repeated adjustments of these weights. One of the important characters of ANNs is that they can learn from their experience in a training environment. The use of multilayer feedforward Perceptron was restricted by the lack of a suitable learning algorithm until Paul Werbos (1974) a PhD student introduced 'backpropagation' learning. ${ }^{9}$ Some of the other popular network designs include Hopfield networks, ${ }_{1}^{10}$ Radial Basis Function ${ }^{11}$ and the Self-Organizing Feature Map. ${ }^{12}$

ANNs have already found a wide variety of applications in the real world. Their ability to classify and recognise patterns accurately has attracted researchers to apply them in solving many clinical problems. As we realise that diagnosis, treatment and predicting outcome in many clinical situations is dependent on a complex interaction of many clinical, biological and pathological variables there is a growing need for analytical tools like ANNs which can exploit the intricate relationships between these variables.

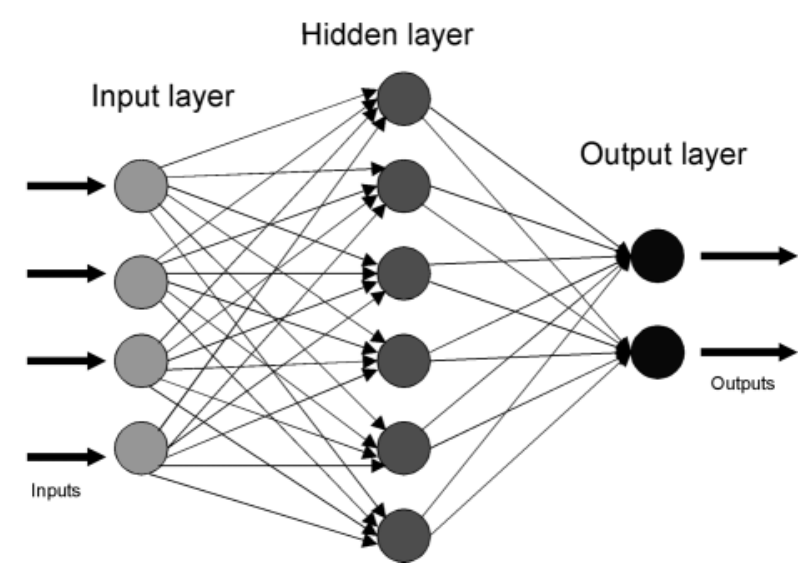

Figure 1 Multilayered feedforward artificial neural networks.

Baxt was one of the first researchers to explore the clinical potentials of ANNs. ${ }^{13}$ He developed a neural network model which accurately diagnosed acute myocardial infarction and latter prospectively validated his work with similar accuracy. ${ }^{14}$ Since then, ANNs have been applied in almost every field of medicine.

\section{Diagnosis}

ANNs have been used in the clinical diagnosis, image analysis in radiology and histopathology, data interpretation in intensive care setting and waveform analysis.

Stamey et al. ${ }^{15}$ developed a neural network derived classification algorithm called ProstAsure Index which can classify prostates as benign or malignant. This model which was subsequently validated in prospective studies had a diagnostic accuracy of $90 \%$, with a sensitivity of $81 \%$ and specificity of $92 \%$. Some of the other surgically relevant diagnostic applications of ANNs include abdominal pain and appendicitis, ${ }^{16}$ retained common bile duct stones, ${ }^{17}$ glaucoma, ${ }^{18}$ and back pain. ${ }^{19}$

ANNs have also been used in diagnosing cytological and histological specimens. PAPNET, a computerised automated screening system based on neural networks, has been developed to assist the cytologist in cervical screening and is one of the few ANN models which was promoted commercially. ${ }^{20}$ Breast, ${ }_{,}^{21}$ gastric ${ }^{22}$ thyroid, ${ }^{23}$ oral epithelial cells, ${ }^{24}$ urothelial cells, ${ }^{25}$ pleural and peritoneal effusion cytology ${ }^{26}$ have all been subjected to analysis by neural networks with varying degree of success. In radiology, it is possible to use both human observations and direct digitised images as inputs to the networks. ANNs have been used to interpret plain radiographs, ${ }^{27}$ ultrasound, ${ }^{28} \mathrm{CT},{ }^{29} \mathrm{MRI},{ }^{30}$ and radioisotope scans. ${ }^{31}$

ANNs pattern recognition ability has been used to analyse various wave forms including the interpretation 


\begin{tabular}{|lll|}
\hline IF & Change in bowel habit OR & Rectal bleeding \\
THEN & Consult your doctor & \\
\hline
\end{tabular}

Figure 2 A typical fuzzy rule system.

of ECGs to diagnose myocardial infarction, ${ }^{32}$ atrial fibrillation, ${ }^{33}$ and ventricular arrythmias. ${ }^{34}$ Analysis of electro-enchalograms (EEG) by neural networks has led to its application in the diagnosis of epilepsy ${ }^{35}$ and sleep disorders. ${ }^{36}$ They have also been trained to analyse electromyographic (EMG) ${ }^{37}$ and Doppler ultrasound ${ }^{38}$ wave forms as well as haemodynamic patterns in intensive care patients. $^{39}$

\section{Prognosis}

Prognostication is extremely important in planning appropriate treatment strategies and follow-up. Accurate identification of high-risk patients may facilitate targeted aggressive adjuvant therapy which may help cure the disease and prolong survival. ANNs with their ability to exploit non-linear relations between variables are particularly suitable to analyse complex cancer data. It has been demonstrated that neural networks can predict survival in patients with breast and colorectal cancer. ${ }^{40,41}$ ANNs have also shown to perform better than consultant colorectal surgeons in predicting outcome in patients with colorectal cancer. ${ }^{42}$ The authors of this paper have demonstrated the generalisability of ANNs, once trained on a particular data, the networks were able to predict outcome for patients from an independent institution with out retraining. ANNs have also been used to predict outcome in lung $^{43}$ and prostate cancers. ${ }^{44}$ They have been applied to predict outcome in intensive care unit and have performed better than APACHE II severity of illness scoring system..$^{45}$

\section{Fuzzy expert systems}

Fuzzy logic is the science of reasoning, thinking and inference that recognises and uses the real world phenomenon - that everything is a matter of degree. Instead of assuming everything is black and white (conventional logic), fuzzy logic recognises that in reality most things would fall somewhere in between, that is varying shades of grey. It was popularised by Lofti Zadeh (1965) an engineer from the University of California. ${ }^{46}$ It uses continuous set membership from 0 to 1 in contrast to Boolean or conventional logic which uses sharp distinctions, i.e. 0 for false and 1 for true. Medicine is essentially a continuous domain and most medical data is inherently imprecise. Fuzzy logic is a data handling methodology that permits ambiguity and hence is particularly suited to medical applications. It captures and uses the concept of fuzziness in a computationally effective manner. Zadeh wrote in 1969 that: 'the most likely area of application for this theory lies in medical diagnostics and, to a lesser extent, in the description of biological systems'. ${ }^{47}$ Fuzzy expert systems have the structure of a series of 'if - then' rules for modelling (Fig. 2).

The techniques of fuzzy logic have been explored in many medical applications. Schneider et al. ${ }^{48}$ showed that fuzzy logic performed better than multiple logistic regression analysis in diagnosing lung cancer using tumour marker profiles. Similarly, the application of fuzzy logic has been explored in the diagnosis of acute leukaemia, ${ }^{49}$ and breast $^{50}$ and pancreatic $c^{51}$ cancer. They have also been applied to characterise ultrasound images of the breast, ${ }^{52}$ ultrasound ${ }^{53}$ and CT scan ${ }^{54}$ images of liver lesions and MRI ${ }^{55}$ images of brain tumours. Fuzzy logic has also been used to predict survival in patients with breast cancer. ${ }^{56}$ Fuzzy controllers have been designed for the administration of vasodilators to control blood pressure in the peri-operative period.$^{57}$ They have also been used for the administration of anaesthetics in the operating room. ${ }^{58}$

\section{Evolutionary computation}

Evolutionary computation is the general term for several computational techniques based on natural evolution process that imitates the mechanism of natural selection and survival of the fittest in solving real-world problems. The most widely used form of evolutionary computation for medical applications are 'Genetic Algorithms'. Proposed by John Holland (1975), ${ }^{59}$ they are a class of stochastic search and optimisation algorithms based on natural biological evolution. They work by creating many random solutions to the problem at hand. This population of many solutions will then evolve from one generation to the next, ultimately arriving at a satisfactory solution to the problem. The best solutions are added to the population while the inferior ones are eliminated. By repeating this process among the better elements, repeated improvements will occur in the population, survive and generate new solutions.

Most medical decisions can be formulated as a search in a very large and complex space. For example: a cytologist analysing a cytological specimen to decide whether they are malignant or not, is searching in the space of all possible cell features for a set of features permitting him to provide a clear diagnosis. Genetic algorithms exploit the mechanism of natural evolution to search efficiently in a given space. They are applied to perform several types of tasks like diagnosis and 
prognosis, medical imaging and signal processing, and planning and scheduling. The principles of Genetic algorithms have been used to predict outcome in critically ill patients, ${ }^{45}$ lung cancer, ${ }^{60}$ melanoma $^{61}$ and response to warfarin. ${ }^{62}$ They have also been used in computerised analysis of mammographic microcalcification, ${ }^{63}$ MRI segmentation of brain tumours to measure the efficacy of treatment strategies ${ }^{64}$ and for analysing computerised 2-D images to diagnose malignant melanomas. ${ }^{63,65}$

\section{Hybrid intelligent systems}

Each AI technique has its own strengths and weaknesses. Neural networks are mainly concerned with learning, fuzzy logic with imprecision and evolutionary computation with search and optimisation. The advantages of these technologies can be combined together to produce hybrid intelligent systems which can work in a complementary manner. Their synergy allows a hybrid system to accommodate common sense, extract knowledge from raw data, use human-like reasoning mechanisms, deal with uncertainty and imprecision, and learn to adapt to a rapidly changing and unknown environment. There are many different hybrid systems available and the popular ones are ANNs for designing fuzzy systems, fuzzy systems for designing ANNs, and Genetic Algorithms for automatically training and generating neural network architectures. Once again, the application of hybrid intelligent systems has been explored in many diverse clinical scenarios. Some examples include breast cancer diagnosis, ${ }^{66}$ analysis of microcalcification on digital mammograms, ${ }^{67}$ diagnosis of coronary artery stenosis, ${ }^{68}$ assessment of myocardial viability, ${ }^{69}$ and control of the depth of anaesthesia. ${ }^{70}$

\section{Conclusions}

There are many different AI techniques available which are capable of solving a variety of clinical problems. However, in spite of earlier optimism, medical AI technology has not been embraced with enthusiasm. One reason for this is the attitude of the clinicians towards technology being used in the decision-making process. Paradoxically, there is no qualm in accepting the biochemical results generated from an auto-analyser or images produced by magnetic resonance imaging. However, it is the obligation of researchers active in this field to produce evidence that these techniques work on a practical level. The need to undertake more randomised controlled studies to prove the efficacy of AI systems in medicine is, therefore, vital.

There is compelling evidence that medical AI can play a vital role in assisting the clinician to deliver health care efficiently in the 21st century. There is little doubt that these techniques will serve to enhance and complement the 'medical intelligence' of the future clinician.

\section{References}

1. Shapiro SC. Artificial intelligence. In: Shapiro SC. (ed) Encyclopedia of Artificial Intelligence, vol. 1, 2nd edn. New York: Wiley, 1992.

2. Turing AM. Computing machinery and intelligence. Mind 1950; 59: 433-60.

3. Lusted LB. Medical progress - medical electronics. N Engl J Med 1955; 252: 580-5.

4. Ledley RS, Lusted LB. Reasoning foundations of medical diagnosis. Science 1959; 130: 9-21.

5. Gunn AA. The diagnosis of acute abdominal pain with computer analysis. J R Coll Surg Edinb 1976; 21: 170-2.

6. Steimann F. On the use and usefulness of fuzzy sets in medical AI. Artif Intell Med 2001; 21: 131-7.

7. McCulloch WS, Pitts W. A logical calculus of the ideas imminent in nervous activity. Bull Math Biophys 1943; 5: 115-33.

8. Rosenblatt F. The Perceptron: a probabilistic model for information storage and organization in the brain. Psychol Rev 1958; 65: 386-408.

9. Werbos P. Beyond regression: new tools for prediction and analysis in the behavioral sciences. PhD Thesis, Harvard University, 1974.

10. Hopfield JJ. Neural networks and physical systems with emergent collective computational abilities. Proc Natl Acad Sci USA 1982; 79: 2554-8.

11. Park J, Sandberg IW. Universal approximation using radial-basis-function networks. Neural Comput 1991; 3: 246-57.

12. Carpenter GA, Grossberg S. The ART of adaptive pattern recognition by a self-organizing neural network. Computer 1988; 21: 77-88.

13. Baxt WG. Use of an artificial neural network for data analysis in clinical decision-making: the diagnosis of acute coronary occlusion. Neural Comput 1990; 2: 480-9.

14. Baxt WG, Skora J. Prospective validation of artificial neural network trained to identify acute myocardial infarction. Lancet 1996; 347: 12-5.

15. Stamey TA, Barnhill SD, Zang Z. Effectiveness of ProstAsure ${ }^{\mathrm{TM}}$ in detecting prostate cancer $(\mathrm{PCa})$ and benign prostatic hyperplasia $(\mathrm{BPH})$ in men age 50 and older. J Urol 1996; 155: 436A.

16. Pesonen E, Ohmann C, Eskelinen M, Juhola M. Diagnosis of acute appendicitis in two databases. Evaluation of different neighborhoods with an LVQ neural network. Methods Inf Med 1998; 37: 59-63.

17. Golub R, Cantu Jr R, Tan M. The prediction of common bile duct stones using a neural network. J Am Coll Surg 1998; 187: 584-90.

18. Henson DB, Spenceley SE, Bull DR. Artificial neural network analysis of noisy visual field data in glaucoma. Artif Intell Med 1997; 10: 99-113.

19. Bounds DG, Lloyd PJ, Mathew BG. A comparison of neural network and other pattern recognition approaches to the diagnosis of low back disorders. Neural Networks 1990; 3: 583-91.

20. Boon ME, Kok LP. Neural network processing can provide means to catch errors that slip through human screening of pap smears. Diagn Cytopathol 1993; 9: 411-6.

21. Downs J, Harrison RF, Kennedy RL, Cross SS. Application of the fuzzy ARTMAP neural network model to medical pattern classification tasks. Artif Intell Med 1996; 8: 403-28.

22. Karakitsos P, Stergiou EB, Pouliakis A, Tzivras M, Archimandritis A, Liossi $\mathrm{AI}$ et al. Potential of the back propagation neural network in the discrimination of benign from malignant gastric cells. Anal Quant Cytol Histol 1996; 18: 245-50.

23. Karakitsos P, Cochand-Priollet B, Guillausseau PJ, Pouliakis A. Potential of the back propagation neural network in the morphologic examination of thyroid lesions. Anal Quant Cytol Histol 1996; 18: 495-500.

24. Brickley MR, Cowpe JG, Shepherd JP. Performance of a computer simulated neural network trained to categorise normal, premalignant and malignant oral smears. J Oral Pathol Med 1996; 25: 424-8.

25. Hurst RE, Bonner RB, Ashenayi K, Veltri RW, Hemstreet 3rd GP. Neural net-based identification of cells expressing the p300 tumor-related antigen using fluorescence image analysis. Cytometry 1997; 27: 36-42.

26. Truong H, Morimoto R, Walts AE, Erler B, Marchevsky A. Neural networks as an aid in the diagnosis of lymphocyte-rich effusions. Anal 
Quant Cytol Histol 1995; 17: 48-54.

27. Ashizawa K, Ishida T, MacMahon H, Vyborny CJ, Katsuragawa S, Doi K Artificial neural networks in chest radiography: application to the differential diagnosis of interstitial lung disease. Acad Radiol 1999; 6: 2-9.

28. Tailor A, Jurkovic D, Bourne TH, Collins WP, Campbell S. Sonographic prediction of malignancy in adnexal masses using an artificial neural network. Br J Obstet Gynaecol 1999; 106: 21-30.

29. Matsuki Y, Nakamura K, Watanabe H, Aoki T, Nakata H, Katsuragawa S et al. Usefulness of an artificial neural network for differentiating benign from malignant pulmonary nodules on high-resolution CT: evaluation with receiver operating characteristic analysis. Am J Roentgenol 2002; 178: 657-63.

30. Lucht R, Delorme S, Brix G. Neural network-based segmentation of dynamic MR mammographic images. Magn Reson Imaging 2002; 20: 147-54.

31. Fisher RE, Scott JA, Palmer EL. Neural networks in ventilation-perfusion imaging. Radiology 1996; 198: 699-706.

32. Heden B, Edenbrandt L, Haisty Jr WK, Pahlm O. Artificial neural networks for the electrocardiographic diagnosis of healed myocardial infarction. Am J Cardiol 1994; 74: 5-8.

33. Yang TF, Devine B, Macfarlane PW. Artificial neural networks for the diagnosis of atrial fibrillation. Med Biol Eng Comput 1994; 32: 615-9.

34. Dassen WR, Karthaus VL, Talmon JL, Mulleneers RG, Smeets JL, Wellens $\mathrm{HJ}$. Evaluation of new self-learning techniques for the generation of criteria for differentiation of wide-QRS tachycardia in supraventricular tachycardia and ventricular tachycardia. Clin Cardiol 1995; 18: 103-8

35. Walczak S, Nowack WJ. An artificial neural network approach to diagnosing epilepsy using lateralized bursts of theta EEGs. J Med Syst 2001; 25: 9-20.

36. Schaltenbrand N, Lengelle R, Toussaint M, Luthringer R, Carelli G, Jacqmin A et al. Sleep stage scoring using the neural network model: comparison between visual and automatic analysis in normal subjects and patients. Sleep 1996; 19: 26-35

37. Abel EW, Zacharia PC, Foster A. Neural network analysis of the EMG interference pattern. Med Eng Phys 1996; 18: 12-7.

38. Smith JH, Graham J, Taylor RJ. The application of an artificial neural network to Doppler ultrasound waveforms for the classification of arterial disease. Int J Clin Monit Comput 1996; 13: 85-91.

39. Spencer RG, Lessard CS, Davilla F, Etter B. Self-organising discovery, recognition and prediction of haemodynamic patterns in the intensive care unit. Med Biol Eng Comput 1997; 35: 117-23.

40. Burke HB, Goodman PH, Rosen DB, Henson DE, Weinstein JN, Harrell Jr FE et al. Artificial neural networks improve the accuracy of cancer survival prediction. Cancer 1997; 79: 857-62.

41. Burke HB, Hoang A, Iglehart JD, Marks JR. Predicting response to adjuvant and radiation therapy in patients with early stage breast carcinoma. Cancer 1998; 82: 874-7.

42. Bottaci L, Drew PJ, Hartley JE, et al. Artificial neural networks applied to outcome prediction for colorectal cancer patients in separate institutions. Lancet 1997; 350: 469-72.

43. Marchevsky AM, Patel S, Wiley KJ, Stephenson MA, Gondo M, Brown RW et al. Artificial neural networks and logistic regression as tools for prediction of survival in patients with stages I and II non-small cell lung cancer. Mod Pathol 1998; 11: 618-25.

44. Han M, Snow PB, Epstein, Chan TY, Jones KA, Walsh PC et al. A neural network predicts progression for men with Gleason score $3+4$ versus $4+3$ tumors after radical prostatectomy. Urology 2000; 56: 994-9.

45. Dybowski R, Weler P, Chang R, Gant V. Prediction of outcome in critically ill patients using artificial neural network synthesised by genetic algorithm. Lancet 1996; 347: 1146-50.

46. Zadeh LA. Fuzzy sets. Inf Control 1965; 8: 338-53.

47. Zadeh LA. Biological application of the theory of fuzzy sets and systems. In: Proceedings of the International Symposium on Biocybernetics of the Central Nervous System. Boston: Little Brown, 1969; 199-212.

48. Schneider J, Bitterlich N, velcovsky HG, Morr H, Katz N, Eigenbrodt E. Fuzzy-logic based tumor-marker profiles improved sensitivity in the diagnosis of lung cancer. Int J Clin Oncol 2002; 7: 145-51.
49. Belacel N, Vincke P, Scheiff JM, Boulassel MR. Acute leukemia diagnosis aid using multicriteria fuzzy assignment methodology. Comput Methods Programs Biomed 2001; 64: 145-51.

50. Sarkar M, Leong TY. Nonparametric techniques to extract fuzzy rules for breast cancer diagnosis problem. Medinfo 2001; 19: 1394-8.

51. Halm U, Rohde N, Klapdor R, Reith HB, Thiede A, Etzrodt Get al. Improved sensitivity of fuzzy logic based tumor marker profiles for diagnosis of pancreatic carcinoma versus benign pancreatic disease. Anticancer Res 2000; 20: 4957-60.

52. Koyama S, Obata $Y$, Shimamoto K, Ishigaki T, Ishii N, Isomoto $Y$ et al. Breast ultrasonography: computer-aided diagnosis using fuzzy inference. J Ultrasound Med 1997; 16: 665-72.

53. Badawi AM, Derbala AS, Youssef AM. Fuzzy logic algorithm for quantitative tissue characterization of diffuse liver diseases from ultrasound images. Int J Med Inf 1999; 55: 135-47.

54. Klein HM, Eisele T, Klose KC, Stauss I, Brenner M, Ameling W et al. Pattern recognition system for focal liver lesions using 'crisp' and 'fuzzy' classifiers. Invest Radiol 1996; 31: 6-10.

55. Fletcher-Heath LM, Hall LO, Goldgof DB, Murtagh FR. Automatic segmentation of non-enhancing brain tumors in magnetic resonance images. Artif Intell Med 2001; 21: 43-63.

56. Seker H, Odetayo MO, Petrovic D, Naguib RNG, Bartoli C, Alasio L et al. Assessment of nodal involvement and survival analysis in breast cancer patients using image cytometric data: statistical, neural network and fuzzy approaches. Anticancer Res 2002; 22: 433-8.

57. Ying H, McEachern M, Eddleman DW. Fuzzy control of mean arterial pressure in postsurgical patients with sodium nitroprusside infusion. IEEE Trans Biomedical Eng 1992; 39: 1060-70.

58. Mason DG, Ross JJ, Edwards ND. Self-learning fuzzy control of atracurium-induced neuromuscular block during surgery. Med Biol Engin Comput 1997; 35: 498-503.

59. Holland JH. Adaptation in Natural and Artificial Systems. Ann Arbor, MI: University of Michigan Press, 1975.

60. Jefferson MF, Pendleton N, Lucas SB, Horan MA. Comparison of a genetic algorithm neural network with logistic regression for predicting outcome after surgery for patients with nonsmall cell lung carcinoma. Cancer 1997; 79: $1338-42$.

61. Sierra B, Larranaga P. Predicting survival in malignant skin melanoma using Bayesian networks automatically induced by genetic algorithms. An empirical comparison between different approaches. Artif Intell Med 1998; 14: 215-30.

62. Narayanan MN, Lucas SB. A genetic algorithm to improve a neural network to predict a patient's response to warfarin. Methods Inf Med 1993; 32: 55-8.

63. Chan HP, Sahiner B, Lam KL. Computerized analysis of mammography microcalcifications in morphological and texture feature spaces. Med Phys 1998; 25: 2007-19.

64. Velthuizen RP, Hall LO, Clarke LP. Feature extraction for MRI segmentation. J Neuroimaging 1999; 9: 85-90.

65. Handels H, Rop T, Kreusch J, Wolff HH, Poppl SJ. Feature selection for optimized skin tumor recognition using genetic algorithms. Artif Intell Med 1999; 16: 283-97.

66. Pena-Reyes, Sipper M. A fuzzy-genetic approach to breast cancer diagnosis. Artif Intell Med 1999; 17: 131-55.

67. Verma B, Zakos J. A computer-aided diagnosis system for digital mammograms based on fuzzy-neural and feature extraction techniques. IEEE Trans Biomedical Eng 2001; 5: 46-54.

68. Sztandera LM, Goodenday LS, Cios KJ. A neuro-fuzzy algorithm for diagnosis of coronary artery stenosis. Comput Biol Med 1996; 26: 97-111.

69. Behloul F, Lelieveldt BP, Boudraa A, Janier MF, Revel D, Reiber JH. Neurofuzzy systems for computer aided myocardial viability assessment. IEEE Trans Med Imaging 2001; 20: 1302-13.

70. Allen R, Smith D. Neuro-fuzzy closed-loop control of depth of anaesthesia. Artif Intell Med 2001; 21: 185-91. 